\title{
Diterpene Ginkgolides Exert an Antidepressant Effect Through the NT3-TrkA and Ras-MAPK Pathways
}

This article was published in the following Dove Press journal: Drug Design, Development and Therapy

\author{
Ting Wang ${ }^{1-3, *}$ \\ Shunjie Bai ${ }^{1-3, *}$ \\ Wei Wang ${ }^{2-4, *}$ \\ Zhi Chen ${ }^{2,3}$ \\ Jianjun Chen $\mathbb{D D}^{2,3}$ \\ Zihong Liang ${ }^{5}$ \\ Xunzhong $\mathrm{Qi}^{2-4}$ \\ Hailan Shen' \\ Peng Xie ${ }^{1-6}$
}

'Department of Laboratory Medicine, The First Affiliated Hospital of Chongqing Medical University, Chongqing, People's

Republic of China; ${ }^{2} \mathrm{NHC}$ Key Laboratory of Diagnosis and Treatment on Brain Functional Diseases, The First Affiliated Hospital of Chongqing Medical University, Chongqing, People's Republic of China;

${ }^{3}$ Chongqing Key Laboratory of

Neurobiology, Chongqing, People's

Republic of China; ${ }^{4}$ Department of

Neurology, The First Affiliated Hospital

of Chongqing Medical University,

Chongqing, People's Republic of China;

${ }^{5}$ Department of Neurology, The Inner

Mongolia Autonomous Region People's

Hospital, Hohhot, Inner Mongolia,

People's Republic of China; ${ }^{6}$ Chongqing

Key Laboratory of Cerebrovascular

Disease Research, Chongqing, People's

Republic of China

*These authors contributed equally to this work

Correspondence: Peng Xie

Department of Neurology, The First

Affiliated Hospital of Chongqing Medical

University, Chongqing, People's Republic

of China

Tel +86-23-68485490

Fax +86-23-68485 III

Email xiepeng@cqmu.edu.cn
Background: Depression is a highly prevalent mental illness that severely impacts the quality of life of affected individuals. Our recent studies demonstrated that diterpene ginkgolides (DG) have antidepressant effects in mice. However, the underlying molecular mechanisms remained much unclear.

Methods: In this study, we assessed the antidepressant effects of chronic DG therapy in rats by evaluating depression-related behaviors, we also examined potential side effects using biochemical indicators. Furthermore, we performed an in-depth molecular network analysis of gene-protein-metabolite interactions on the basis of metabolomics.

Results: Chronic DG treatment significantly ameliorated the depressive-like behavioral phenotype. Furthermore, the neurotrophin signaling-related NT3-TrkA and Ras-MAPK pathways may play an important role in the antidepressant effect of DG in the hippocampus.

Conclusion: These findings provide novel insight into the mechanisms underlying the antidepressant action of DG, and should help advance the development of new therapeutic strategies for depression.

Keywords: diterpene ginkgolides, antidepressant, neurotrophin, hippocampus, NT3-TrkA and Ras-MAPK pathways

\section{Introduction}

Depression is a chronic mental disease that is a serious threat to human health. ${ }^{1-3}$ Current research into depression is mainly focused on molecular perturbations in the brain, including monoamine neurotransmitter deficiency, oxidative stress disorder and neurogenic disorder. However, available antidepressants benefit fewer than $50 \%$ of patients..$^{4-7}$ Our recent studies demonstrated that diterpene ginkgolides (DG), the main components of ginkgo biloba extract, have antidepressant effects. $^{8-10}$

DG is natural platelet activating factor receptor antagonists that effectively inhibit thrombosis and protect against cerebral ischemia and brain inflammation. Studies show that DG has antioxidant, anti-inflammatory and anti-apoptotic effects, which protects brain neurons and improves brain functions, including cognition. ${ }^{11-13}$ Our previous studies demonstrated that DG significantly improved the depression-like behavioral phenotypes associated with anhedonia, behavioral despair and social disorders in mice. ${ }^{8-10}$ Furthermore, initial studies suggest that DG exerts antidepressant effects by ameliorating brain neurotransmitter levels and metabolic disorders. However, the underlying molecular mechanisms are still unclear, and long-term 
changes in liver and kidney function, blood sugar and blood lipids have not been reported.

Nt3-TrkA is a signaling pathway associated with the neurotrophin family, and plays an important role in axonal growth. ${ }^{14,15}$ The Ras-MAPK signaling pathway plays a key role in neuronal plasticity, oxidative stress, and also participates in regulating various physiological processes such as cell growth, differentiation and apoptosis. ${ }^{16,17}$ NT3-TrkAdependent axonal growth is associated with activation of the Ras-MAPK signaling pathway. ${ }^{18,19}$ Many studies have shown that the NT3-TrkA and RAS-MAPK pathways are closely related to the pathogenesis and treatment of depression. $^{20-23}$ However, whether DG exert antidepressant effects through these two pathways has not been reported.

To clarify the molecular mechanisms by which DG exerts its antidepressant effects, we combined molecular biology and bioinformatics on the basis of metabolomics. We performed an in-depth molecular network analysis of gene-protein-metabolite interactions for the differential metabolites. Based on our previous study, ${ }^{10}$ we selected two brain regions of the hippocampus (HP) and prefrontal cortex (PFC) to compare the antidepressant effects of DG for the analyses. The hippocampus, an important emotional center, is associated with various emotional disorders, and is often used to examine the effects of psychoactive drugs. ${ }^{24,25}$ The PFC, which is associated with emotion, cognition and learning, plays an important role in the pathogenesis of depression. ${ }^{26,27}$ Our previous studies revealed neurobiological changes and metabolic dysfunction in the $\mathrm{HP}^{28,29}$ as well as the $\mathrm{PFC}^{30,31}$ in the rat depression model.

We used depression-related behavioral tests to evaluate the antidepressant-like effect of DG on rats. We also examined potential side effects using biochemical indicators in serum. The non-targeted metabolomics approach, based on gas chromatography-mass spectrometry (GC-MS), was used to obtain total metabolic data for the HP and PFC. Molecular interaction network and pathway analyses of the differential metabolites were carried out on the Ingenuity Pathways Analysis (IPA) platform. Quantitative real time polymerase chain reaction (RT-qPCR) and Western blotting were performed to verify the affected pathways.

\section{Materials and Methods}

\section{Animals and Ethics Statement}

Healthy male Sprague-Dawley rats (age, 10 weeks; weight, approximately $400 \mathrm{~g}$ ) were purchased from the experimental animal center of Chongqing Medical University (China). During the research period, the rats were kept in a stable environment, with lights on 07:00-19:00, temperature of $21-22^{\circ} \mathrm{C}$, and a relative humidity of $55 \pm 5 \%$. All animal experiments were approved by the Ethics Committee of Chongqing Medical University (permit number: 20120126), and were in accordance with the Guide for the Care and Use of Laboratory Animals.

\section{Reagents and Experimental Groupings}

DG Meglumine Injection was a commercial drug of diterpene ginkgolides, ${ }^{9,10}$ which was provided by Jiangsu Kanion Pharmaceutical Co., Ltd (Shanghai, China). It is a mixture of different diterpene ginkgolides (mainly contains ginkgolide A, B and K), which are responsible for the main pharmacological neuroprotective in ginkgo biloba extract. ${ }^{10}$

After 1 week of adaptation, the rats were randomly divided into control (CON) and DG groups. The CON group $(n=15)$ received intraperitoneal injection of $0.9 \%$ $\mathrm{NaCl}$ solution daily, and the DG group $(\mathrm{n}=15)$ received DG Meglumine Injection $(5.4 \mathrm{mg} / \mathrm{kg}$, diluted in $0.9 \%$ $\mathrm{NaCl}$ solution) daily. The choice of drug concentration was based on our previous study. ${ }^{8,10}$ The drug treatment lasted 4 weeks until brain tissue was collected.

\section{Behavioral Tests}

After 4 weeks of drug treatment, behavioral experiments were conducted (Figure 1). All behavioral data were analyzed with an animal trajectory analysis software (SMART, Panlab SL, Barcelona, Spain).

The open field test (OFT) was used to assess the locomotor and anxiety-like behavior of rats. ${ }^{30}$ The open field box was composed of an enclosed open square area, $100 \mathrm{~cm} \times 100 \mathrm{~cm}$, with 40-cm-high walls. During the behavioral test, each rat was placed in the open field box and allowed to move freely for $5.5 \mathrm{~min}$. The total distance (movement) was calculated to evaluate locomotor activity. The number into the center zone and the distance in the center zone were calculated to evaluate anxiety behavior. The more number entries and distance in the center zone, the less anxious behavior is represented in the rat.

The tail suspension test (TST) $)^{32,33}$ and forced swimming test $(\mathrm{FST})^{34}$ were both used to assess behavioral despair. The immobility time can reflect the degree of despair. The longer the immobility time, the greater the despair. The TST was carried out as previously described. ${ }^{32,33}$ Briefly, rats were suspended in a black box $(30 \mathrm{~cm} \times 30 \mathrm{~cm}$; height, $53 \mathrm{~cm})$ by a small metal hook fixed with adhesive tape wrapped 
Treatment (i.p.) (DG or vehicle)

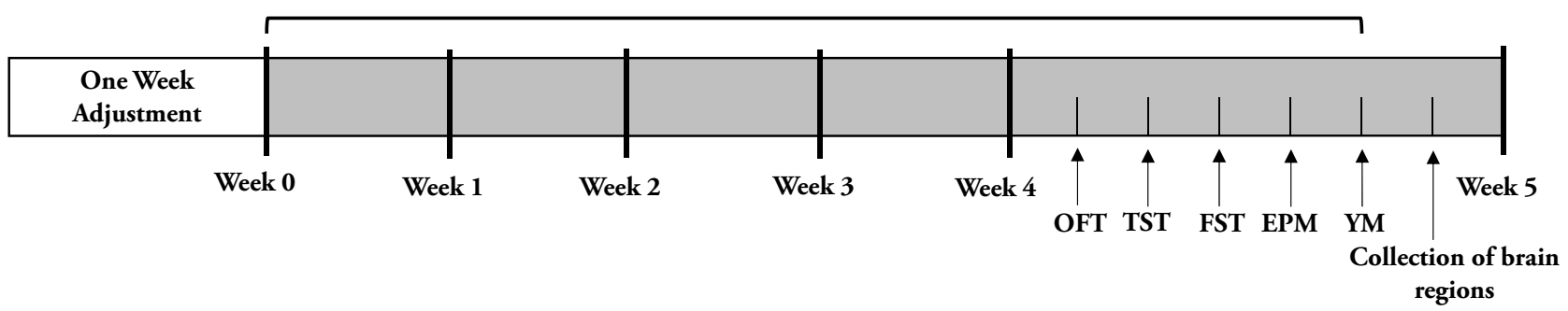

Figure I Schematic schedule of experimental approach.

around the tail. Every test session lasted $6 \mathrm{~min}$ and the last 5 min were scored by SMART for the immobility of rats. Rats were returned to their home cages after test. Animals were considered to be immobile only when they were hung on the hooks passively and completely motionless.

The FST was conducted in a clear Plexiglas cylinder (diameter, $20 \mathrm{~cm}$; height, $40 \mathrm{~cm}$ ) filled with $30 \mathrm{~cm}$ height of water $\left(25 \pm 1{ }^{\circ} \mathrm{C}\right)$. Video was recorded for $6 \mathrm{~min}$, and the final 5 min was used for analysis. Rats were considered to be immobile only with occasional slight movements which were required to keep the mouse's head out of water.

The elevated plus maze (EPM) is used to evaluate anxiety, and is based on the natural aversion of rodents for open and elevated areas, as well as on their innate exploratory behavior in novel environments. The maze consisted of two open arms and two closed arms (length, $50 \mathrm{~cm}$; width, $10 \mathrm{~cm}$ ). Videos were collected for $5.5 \mathrm{~min}$. The last $5 \mathrm{~min}$ were used to calculate the percentage distance traveled and the time spent in the open arms.

The Y-maze (YM) was used to study the spatial recognition and memory ability. It consisted of three identical arms (length, $45 \mathrm{~cm}$; width, $10 \mathrm{~cm}$; height, $29 \mathrm{~cm}$ ). Rats were placed at the end of an arm, and the order in which they entered each arm within an 8-min period was recorded. Alternation was defined as consecutive entry into three different arms (such as 1, 2, 3 or 1, 3,2). The maximum alternation was the total number of arm entries minus 2, and the percentage was defined as the actual alternation/maximum alternation $\times 100 \%{ }^{35}$

\section{Brain Tissue and Serum Preparation}

After the behavioral tests were completed, the brain tissue and serum were collected. The rats were anesthetized with $50 \mathrm{mg} / \mathrm{kg}$ pentobarbital sodium, and blood was collected from the heart. The rats were then decapitated, and the brain was removed from the skull. The HP and PFC were isolated, quickly frozen in liquid nitrogen, and stored at $-80^{\circ} \mathrm{C}$ until analyses. The serum was separated from whole blood after centrifugation for $10 \mathrm{~min}\left(3000 \mathrm{rpm}, 4{ }^{\circ} \mathrm{C}\right)$.

\section{Biochemical Indicator Analysis}

Serum indicators of hepatic function (ALT, alanine aminotransferase; AST, aspartate aminotransferase; TBA, total bile acid; TBil, total bilirubin), renal function (Crea; Urea; UA, uric acid), lipids (TC, total Cholesterol; TG, triglyceride; HDL-C, high-density lipoprotein cholesterol; LDL-C, low-density lipoprotein cholesterol), glucose and homocysteine (HCY) were measured using commercially available enzymatic colorimetric assays and an automated analyzer system (Cobas 8000 modular device Roche Diagnostics, Switzerland).

\section{GC-MS Analysis}

The processing of GC-MS samples was according to previous publications from our laboratory. ${ }^{36-38}$ The tissue samples, containing an internal standard (2-chloroL-phenylalanine, $75 \mathrm{ng} / \mathrm{mL}$ ), were thoroughly mixed with a methanol-water solution $(4 / 1, \mathrm{v} / \mathrm{v})$ to $600 \mu \mathrm{L}$. The mixture was sonicated for $10 \mathrm{~min}$ and then centrifuged for $10 \mathrm{~min}$ $\left(14,000 \mathrm{rpm}, 4^{\circ} \mathrm{C}\right)$. The supernatant was collected, transferred to a glass derivatization vial, and concentrated for rapid enrichment. After the liquid was fully volatilized, $80 \mu \mathrm{L}$ methoxamine hydrochloride in pyridine $(15 \mathrm{mg} / \mathrm{mL})$ was added to the vial. The sample was then vortexed for $2 \mathrm{~min}$, and incubated for $90 \mathrm{~min}$ at $37^{\circ} \mathrm{C}$. Finally, $80 \mu \mathrm{L}$ BSTFA (including 1\% TMCS) was added. After 2 min of vortexing, the mixture was incubated for $60 \mathrm{~min}$ at $70^{\circ} \mathrm{C}$, and then at room temperature for $30 \mathrm{~min}$. Non-targeted GC-MS metabolomic analysis was carried out on these samples on the Agilent 7890A/5975C GC/MSD system (splitless mode; Agilent Technologies, Santa Clara, California, USA) using an Agilent J\&W HP-5ms capillary column $(30 \mathrm{~m} \times 0.25 \mathrm{~mm}$ $\times 0.25 \mu \mathrm{m})$. The corresponding detection parameters were as follows (helium as carrier gas, purity $99.999 \%$, constant flow 
rate of $6 \mathrm{~mL} / \mathrm{min}$ ): initial temperature of $70{ }^{\circ} \mathrm{C}$ for $2 \mathrm{~min}$, increased to $160{ }^{\circ} \mathrm{C}$ at a rate of $6^{\circ} \mathrm{C} / \mathrm{min}$, increased to $240{ }^{\circ} \mathrm{C}$ at a rate of $10{ }^{\circ} \mathrm{C} / \mathrm{min}$, increased to $300{ }^{\circ} \mathrm{C}$ at a rate of $20{ }^{\circ} \mathrm{C} / \mathrm{min}$, and then maintained at $300{ }^{\circ} \mathrm{C}$ for $6 \mathrm{~min}$. The full scanning mode was used to detect the mass spectrum, and the range of mass spectrometry detection was 50-600 $(\mathrm{m} / \mathrm{z})$.

\section{Metabolomics Data and Bioinformatics Analysis}

The original GC-MS data were converted into NetCDF format using TagFinder software. ${ }^{39}$ Mass spectrum data preprocessing was carried out using $\mathrm{R}$ software, including peak recognition, peak alignment and retention time correction. The final data editing was carried out in MS EXCEL 2010 software to obtain the final 2D data matrix, including sample information, retention time and mass spectrum response intensity. The peak area normalization method was used to normalize the mass spectrum response intensity, and the normalized results were imported into SIMCA-P 14.0 software (MKS Umetrics AB) for multivariable statistical analysis, including pair-wise orthogonal projections to latent structures-discriminant analyses (OPLS-DA).

The OPLS-DA method was used to eliminate the variability of non-related metabolites and to identify the most predictive metabolites between the different groups. RPT was used to validate the results of the OPLS-DA analysis with 200-iteration permutations. In the OPLS-DA model, candidate metabolites were selected based on the threshold of variable importance in the projection value (VIP > 1.0). Furthermore, univariate statistical analysis of the identified metabolites was performed using Student's $t$-test.

\section{Biological Functions and Pathways Analysis}

MetaboAnalyst 4.0 (http://www.metaboanalyst.ca) was used to generate heat maps of the differential metabolites. ${ }^{40}$ The differential metabolites were subjected to metabolite set enrichment analysis (MSEA) and pathway analysis using MetaboAnalyst 3.0. IPA software was used for biological functions pathway analysis of the differential metabolites in the HP or PFC, and the network score was used to determine the relevance between the metabolites and networks. The metabolites, including KEGG IDs, fold-changes and $P$-values, were uploaded to the Ingenuity Pathways Analysis server (IPA, Qiagen, Redwood City, CA, USA). ${ }^{41}$ Highly correlated molecular and cellular functions were determined using the Ingenuity Pathway Knowledge
Base, and Cytoscape 3.4.0 was used to build the network diagram of the differential metabolites and molecular functions in the HP or PFC. ${ }^{40}$ Correlation analyses of behavior, genes and metabolites were performed using Pearson's correlation coefficient.

\section{RT- qPCR Analysis and Western Blotting Validation}

RT-qPCR was used to assess mRNA expression of components of the Ras-MAPK and NT3-TrkA pathways. The synthesis of cDNA from the HP or PFC was carried out using the PrimeScript RT reagent Kit (TAKARA), and DNA amplification was performed with a SYBR green detection system (Roche, Germany). The housekeeping gene $\beta$-actin was used to normalize the data, and data analysis was performed with the $2^{(-\Delta \Delta \mathrm{CT})}$ method. The primers are shown in Table S1. The HP and PFC brain tissue from the DG and CON groups ( $n=6$ per group) were fully lysed in RIPA buffer containing phosphatase inhibitor (Roche, Germany) at $4{ }^{\circ} \mathrm{C}$. The proteins were separated on a 10 $-12 \%$ SDS-PAGE gel and transferred to a PVDF membrane (Millipore, Billerica, MA, USA) by semi-dry transfer. After blocking with $5 \%$ skim milk, the membranes were incubated with rabbit polyclonal anti-Raf (Ruiying Bio; 1:2000), rabbit polyclonal anti-NT3 (Abcam; 1:1000), rabbit monoclonal anti-Ras (Beyotime; 1:5000), rabbit monoclonal anti-TrkA (Beyotime; 1:2000) or mouse monoclonal anti-GAPDH (Bio-Rad; 1:12,000) overnight at $4{ }^{\circ} \mathrm{C}$. After washing, the blots were incubated with secondary antibodies at room temperature for $2 \mathrm{hrs}$. Finally, the signals were visualized using an ECL kit (Millipore).

\section{Statistical Analysis}

Statistical analyses were performed using Graphpad Prism 7.0 software (IBM). Two-tailed $t$-tests were used to compare the CON and DG groups, and data were expressed as mean \pm standard error of the mean (SEM).

\section{Results}

\section{Effects of DG on Behavior}

After 4 weeks of DG treatment, there was a significant difference in the number of entries and the distance traveled in the central area in the OFT compared with the CON group (Figure 2A and B). This indicates that DG treatment significantly reduces anxiety-related behavior in rats. However, the treatment of DG did not affect the total locomotor activity in the open field test (Figure S1). In addition, the immobility 

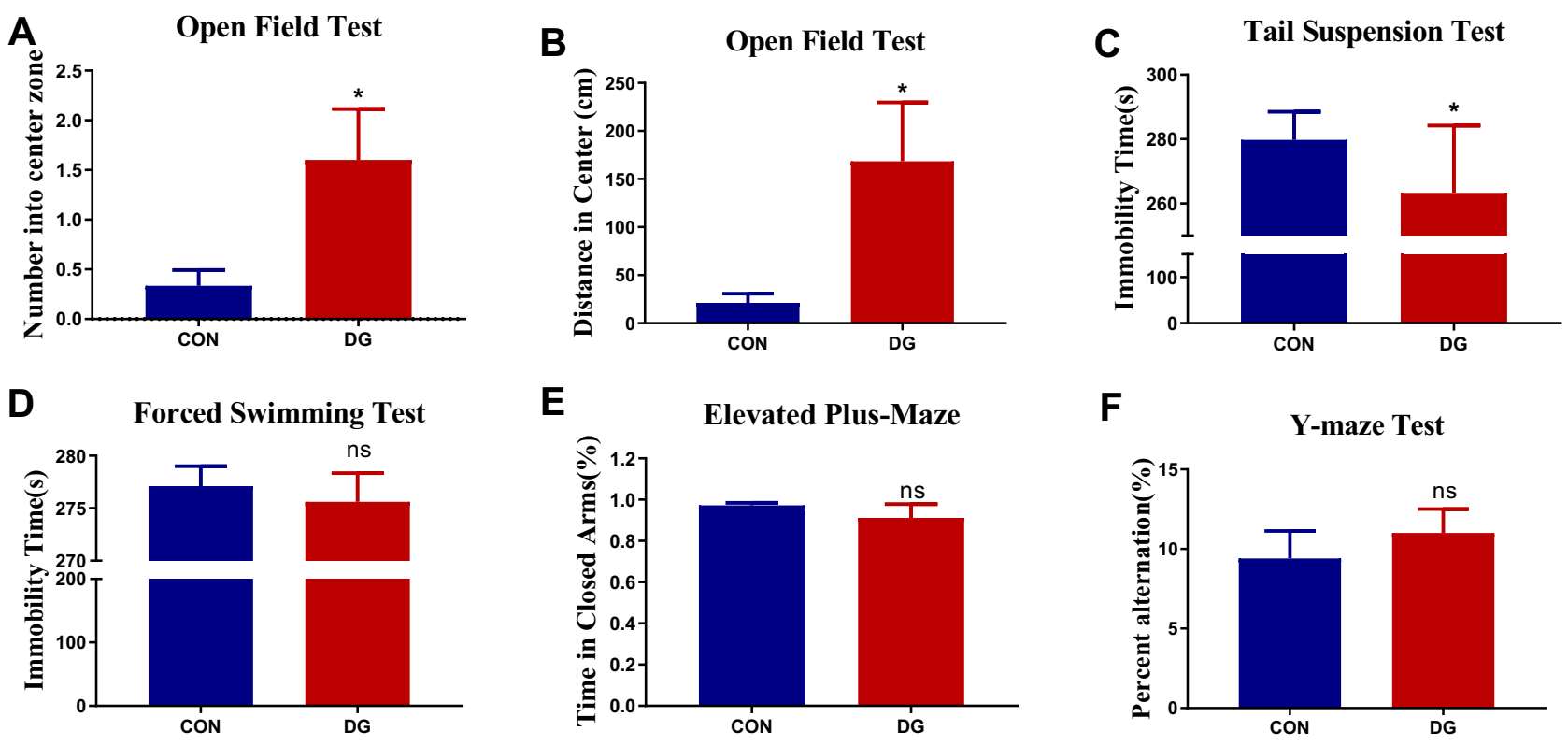

Figure 2 Effects of DG treatment on performance in behavioral tests. (A) The number of entries in the central area in the open field test (OFT). (B) The distance traveled in the central area in the OFT. (C) Immobility time in the tail suspension test (TST). (D) Immobility time in the forced swimming test (FST). (E) The percentage of time spent in the closed arm in the elevated plus-maze (EPM). (F) The percent alternation in the Y-maze test after 4 weeks of DG treatment. Values represent the mean \pm SEM ( $n=15$ rats per group). $* p<0.05, \mathrm{~ns}=$ not significant, compared with the control $(\mathrm{CON})$ group.

time was substantially less in the DG group compared with the CON group in the TST (Figure 2C), indicating that DGs have an antidepressant-like effect. However, in the FST, only a downward trend was observed (Figure 2D). Similarly, DG treatment had a slight, but non-significant, effect in both the EPM and the YM (Figure 2E and F), indicating that DGs may not improve spatial memory in rats.

\section{Effects of DG on Biochemical Indicators}

To assess whether long-term DG treatment has side effects, we tested for biochemical indicators of physiological function. After 4 weeks of DG treatment, there was no significant difference in biochemical indicators of hepatic function, renal function, lipids, glucose or HCY compared with the normal control group (Table 1). These results show that chronic DG treatment effectively attenuates despair and anxiety-like behavior in rats without negatively affecting systemic functions.

\section{GC-MS Metabolomics Analysis}

OPLS-DA dimension reduction analysis was carried out, and the results were discriminated and analyzed in combination with the regression model. This revealed a significant difference between the $\mathrm{CON}$ and $\mathrm{DG}$ groups. The OPLS-DA score plots for the HP $\left(R^{2} \mathrm{X}=0.377, \mathrm{R}^{2} \mathrm{Y}=\right.$ $0.963, \mathrm{Q}^{2}=0.795$, Figure $\left.3 \mathrm{~A}\right)$ and the PFC $\left(\mathrm{R}^{2} \mathrm{X}=0.269\right.$,
$\mathrm{R}^{2} \mathrm{Y}=0.81, \mathrm{Q}^{2}=0.142$, Figure $3 \mathrm{~B}$ ) showed that this model was robust and reliable.

\section{Screening of Differential Metabolites}

The differential metabolites were screened according to the set criteria (VIP $>1$ and FDR $<0.05$ ) based on multivariable statistics and univariate statistics. A total of 29 differential metabolites were selected for the HP (Table S2; Figure 3C), including 7 downregulated metabolites and 22 upregulated metabolites, and a total of 16 differential metabolites were selected for the PFC (Table S3; Figure 3C), including 5 downregulated metabolites and 11 upregulated metabolites. Among the differential metabolites, 2 (phosphate and mono(2-ethylhexyl)phthalate) were altered both in the HP and the PFC (Figure 3C). The heat maps for the HP and PFC are shown in Figure 4.

\section{Enrichment Analysis and Pathway Analysis} MetaboAnalyst, employing the KEGG metabolite library, was used for functional cluster and pathway analysis of the differential metabolites. According to the screening criteria (false discovery rate $(\mathrm{FDR})<0.05$ and impact value $>0$ ), functional enrichment analysis of the HP differential metabolites was performed (Figure 5A). Pathway analysis revealed that the metabolites were mainly related to betaalanine metabolism and arginine and proline metabolism 
Table I Key Biochemical Indicators in the Comparison of Diterpene Ginkgolides (DG) and Control (CON) Groups in Rat Serum

\begin{tabular}{|c|c|c|c|c|}
\hline Category & Biochemical Indicators & CON (Mean士SEM) & DG (Mean \pm SEM) & T-test (p) \\
\hline \multirow[t]{4}{*}{ Hepatic function } & $\mathrm{ALT}(\mathrm{U} / \mathrm{L})$ & $46.36 \pm 3.12$ & $45.13 \pm 2.42$ & 0.759 \\
\hline & $\mathrm{AST}(\mathrm{U} / \mathrm{L})$ & $91.79 \pm 7.28$ & $106.33 \pm 6.72$ & 0.153 \\
\hline & $\mathrm{TBA}(\mu \mathrm{mol} / \mathrm{L})$ & $11.09 \pm 1.63$ & $8.15 \pm 1.08$ & 0.139 \\
\hline & $\operatorname{TBil}(\mu \mathrm{mol} / \mathrm{L})$ & $0.66 \pm 0.08$ & $0.56 \pm 0.06$ & 0.334 \\
\hline \multirow[t]{3}{*}{ Renal function } & Crea $(\mu \mathrm{mol} / \mathrm{L})$ & $36.07 \pm 2.01$ & $40.4 \pm 2.14$ & 0.153 \\
\hline & Urea $(\mathrm{mmol} / \mathrm{L})$ & $7.66 \pm 0.3$ & $7.09 \pm 0.2$ & 0.124 \\
\hline & $\mathrm{UA}(\mu \mathrm{mol} / \mathrm{L})$ & $48.07 \pm 5.03$ & $45.53 \pm 2.48$ & 0.656 \\
\hline \multirow[t]{4}{*}{ Lipids } & $\mathrm{TC}(\mathrm{mmol} / \mathrm{L})$ & $1.36 \pm 0.05$ & $1.29 \pm 0.05$ & 0.339 \\
\hline & $\mathrm{TG}(\mathrm{mmol} / \mathrm{L})$ & $1.01 \pm 0.11$ & $1.05 \pm 0.09$ & 0.752 \\
\hline & $\mathrm{HDL}-\mathrm{C}(\mathrm{mmol} / \mathrm{L})$ & $0.96 \pm 0.06$ & $0.96 \pm 0.04$ & 0.922 \\
\hline & LDL-C(mmol/L) & $0.27 \pm 0.02$ & $0.23 \pm 0.02$ & 0.243 \\
\hline \multirow[t]{2}{*}{ Others } & $\mathrm{Glu}(\mathrm{mmol} / \mathrm{L})$ & $14.86 \pm 0.76$ & $|7.1| \pm 0.99$ & 0.085 \\
\hline & $\mathrm{HCY}(\mu \mathrm{mol} / \mathrm{L})$ & $10.4 \pm 0.7$ & $11.45 \pm 0.39$ & 0.187 \\
\hline
\end{tabular}

Abbreviations: ALT, alanine aminotransferase; AST, aspartate aminotransferase; TBA, total bile acid; TBil, total bilirubin; UA, uric acid; TC, total Cholesterol; TG, triglyceride; HDL-C, high-density lipoprotein cholesterol; LDL-C, low-density lipoprotein cholesterol; Glu, glucose; HCY, homocysteine.

(Figure 5B). In contrast, functional enrichment of the PFC showed that the differential metabolites were primarily related to aspartate metabolism, phenylalanine and tyrosine metabolism and urea cycle (Figure 5C). Pathway analysis showed that they were mainly related to aminoacyl-tRNA biosynthesis, galactose metabolism and phenylalanine, tyrosine and tryptophan biosynthesis (Figure 5D).

\section{Metabolic Network Interaction and Functional Prediction}

The differential metabolites for the HP and PFC were uploaded to the IPA analysis platform for metabolic network interaction and molecular and cellular functional analysis. The top five functions of the HP metabolites were cellular function and maintenance, molecular transport, small molecule biochemistry, amino acid metabolism, and cell death and survival. The top five functions of the PFC metabolites were cellular growth and proliferation, cell death and survival, amino acid metabolism, lipid metabolism, and molecular transport (Table S4, Figure 6A and B). Network functions of "carbohydrate metabolism, molecular transport, small molecule biochemistry" were associated with the key differential metabolites in the HP with a score of 20 and 8 differential metabolites involved (Figure 7A). Moreover, the network of "cell death and survival, cellular growth and proliferation, organismal development" was associated with the key differential metabolites in the PFC, with a score of 28 and 10 differential metabolites involved (Figure 7B). The MAPK pathway was associated with the metabolic network interaction of the HP and PFC. By searching the KEGG pathway database, two closely related MAPK signaling pathways were found: NT3-TrkA and Ras-MAPK pathways.

\section{Relative mRNA Expression Level}

The expression levels of NGF, NT3, TrkA, Crk, C3G, Rap1, B-Raf, MEK1, MEK2, Erk1 and Erk2 in the NT3TrkA pathway and of TrkB, Ras, Raf, MEK1, MEK2, Erk1 and Erk2 in the Ras-MAPK pathway are shown in Figure 8. In the NT3-TrkA pathway, the expression levels of NT3 and TrkA in the HP and PFC were significantly upregulated, and other related genes showed an upward trend (Figure 8A and B). However, in the Ras-MAPK pathway, there was no significant difference in gene expression compared with the control group in the HP or PFC (Figure 8C and D). Interestingly, the two trends were not consistent. In the HP, Ras-MAPK signaling pathway-related genes were substantially upregulated, while in the PFC, there was no significant change in expression levels. This indicates that DG may exert an anti-depressive effect by activating the NT3TrkA and Ras-MAPK pathways in the HP, or by activating NT3-TrkA or other signaling pathways in the PFC.

\section{Western Blotting}

Four key proteins in the NT3-TrkA and Ras-MAPK pathways were subjected to Western blotting verification (Figure 9). In the HP, the protein levels of NT3, TrkA, Ras and Raf were significantly increased compared with 

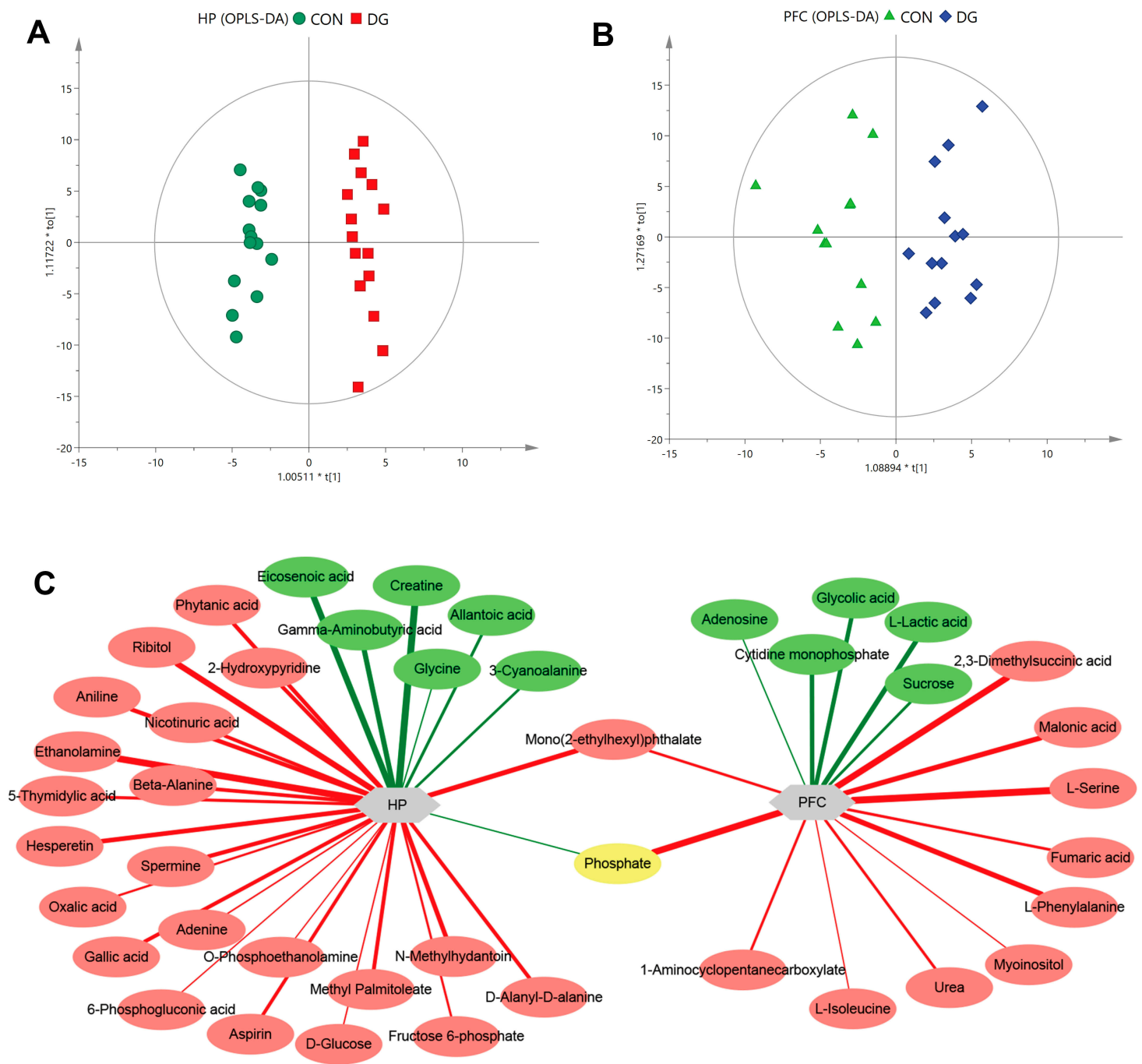

Figure 3 Multivariate statistical analysis. OPLS-DA score plot derived from GC-MS analysis between CON and DG groups in the hippocampus (HP) (A) and prefrontal cortex (PFC) (B). Network analysis of metabolites altered in the HP or PFC of rats treated with DG for 4 weeks (C). Red-colored boxes and red-colored lines indicate upregulation, while green-colored boxes and green-colored lines indicate downregulation. The line widths represent the VIP scores of differential metabolites.

the control group (Figure 9A), while only TrkA showed a significant difference in the PFC (Figure 9B).

\section{Discussion}

Our previous studies showed that DG ameliorated brain neurotransmitter perturbation and metabolic dysfunction in mice. ${ }^{8-10}$ However, the underlying molecular mechanisms, including gene-protein-metabolite interactions, remained unclear. Here, we used metabolomics combined with molecular biology methods to explore the antidepressant mechanisms of DG in the HP and PFC. This revealed that the antidepressant effect of DG involved activation of the neurotrophic NT3-TrkA pathway and the neural plasticityrelated Ras-MAPK pathway in the HP.
The OFT is commonly used to evaluate anxietyrelated behavior in rats. ${ }^{42}$ The number of entries and distance traveled in the central area of the open field were significantly higher compared with the $\mathrm{CON}$ group, which indicated that chronic DG therapy effectively attenuated anxiety behaviors in the rats. The TST and FST are widely used to assess behavior despair in rodent animals. ${ }^{43,44}$ In this study, the immobility time of TST was significantly shorter in the DG group than in the control group, indicated that DG effectively attenuated despair behaviors in the rats. While in the FST, it only exhibited a downward trend. The discrepancy may be related to differences in experimental conditions. The EPM is used to evaluate the anxiety-like behavior of animals, and the Y-maze is used to evaluate learning and 
A

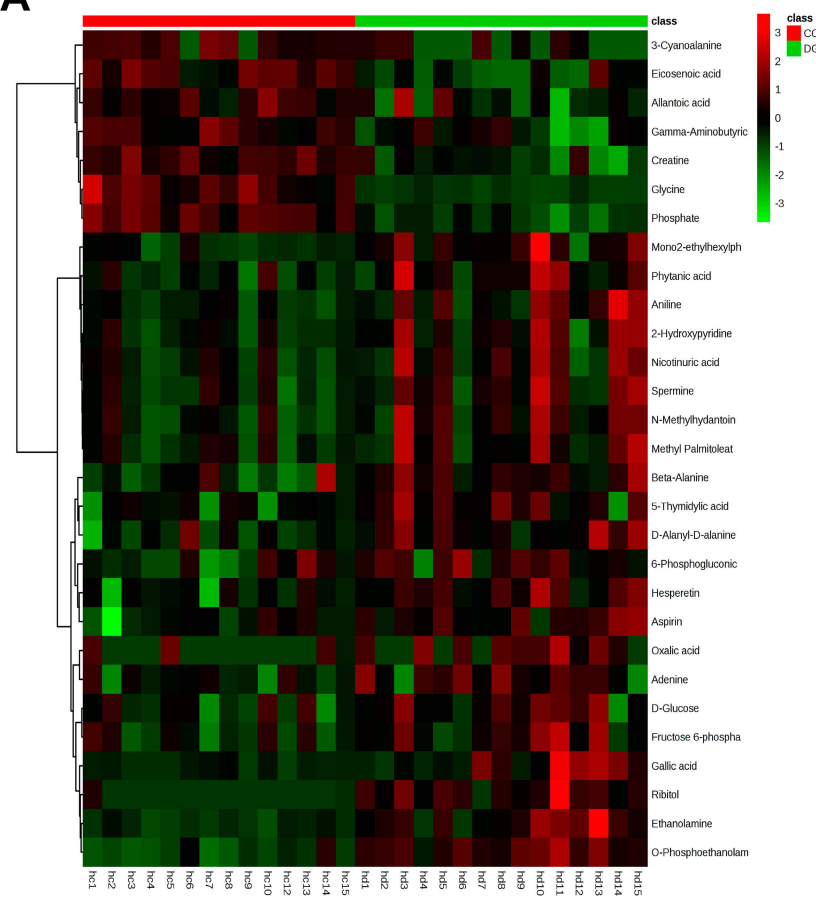

B

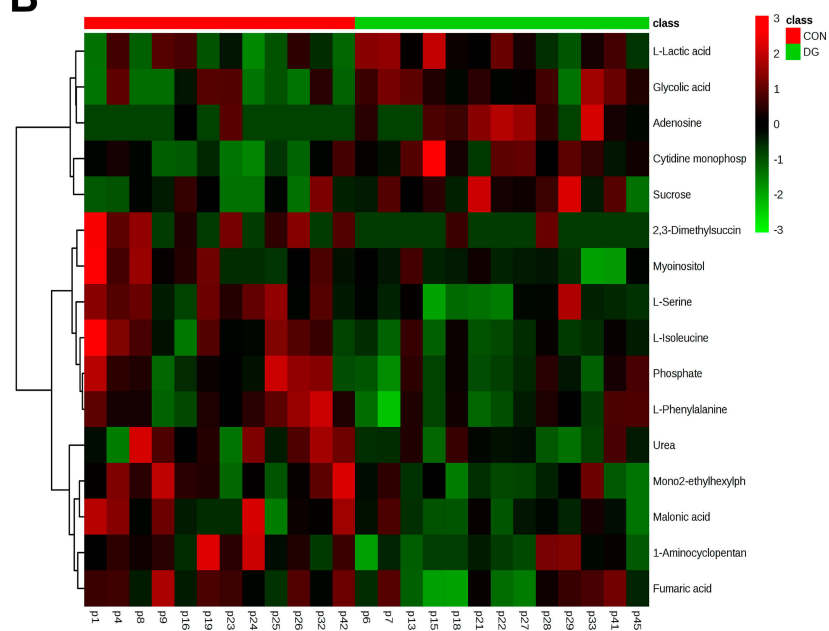

Figure 4 Heat map of the differential metabolites between DG and CON groups in the HP (A). Heat map of the differential metabolites between DG and CON groups in the PFC (B).

memory. ${ }^{45}$ Compared with the control group, there was no significant difference in these behavioral experiments. This indicates that DG have no effect on learning and memory functions in the rat. Therefore, chronic DG therapy effectively attenuated anxiety and depression-related behaviors in the rats.

Then, this study examined whether the therapy of DG had side effects. We accordingly tested for biochemical indicators of hepatic function, renal function, lipids, glucose and HCY. These indicators are the main indices of systemic physiological function. We found no significant change in liver or kidney function in the DG group compared with the control group. There were also no significant changes in blood glucose or lipids in the DG group. There is evidence from laboratory and clinical studies that HCY has direct toxic effects on both the vascular and nervous systems. ${ }^{46}$ We observed no change in HCY after DG treatment. Together, these findings suggest that DG has no neurotoxic or systemic side effects.

GC-MS-based metabolomics identified 29 differential metabolites in the HP and 16 differential metabolites in the PFC. Most of these were upregulated, but only two (phosphate and mono(2-ethylhexyl)phthalate) were simultaneously upregulated in both the HP and PFC. This suggests that the antidepressant-like effect of DGs is not associated with identical metabolic changes in the HP and PFC.

Phosphate is the substrate for the phosphorylation of ADP to ATP in oxidative phosphorylation. ${ }^{47}$ In the regulation of oxidative phosphorylation, phosphate is a putative cytosolic signaling molecule. ${ }^{48}$ Using energy from glucose metabolism, phosphate and ADP produce ATP through mitochondrial oxidative phosphorylation. ${ }^{49}$ This suggests that, to some extent, phosphate and glucose metabolism are positively correlated. A study showed that glucose metabolism in the dorsal structure decreased, while it increased in the ventral structure, following antidepressant treatment. ${ }^{50}$ This is similar to the location of the HP and PFC in rats. Consistent with this study, we found here that phosphate was downregulated in the HP and upregulated in the PFC, suggesting that DG exert an anti-depressive effect by affecting both the HP and the PFC. The converse changes in phosphate may be related to differences in the executive functions of the HP and PFC. . $^{51,52}$

Mono(2-ethylhexyl)phthalate is an environmental endocrine disruptor with a structure similar to tryptophan metabolites. ${ }^{53}$ Studies have shown that this metabolite reversibly inhibits $\alpha$-amino- $\beta$-carboxymuconate- $\varepsilon$-semialdehyde decarboxylase (ACMSD) during tryptophan metabolism, thereby affecting the formation of $\alpha$-aminomuconate- $\varepsilon$ semialdehyde (AMS). ${ }^{54}$ Activation of ACMSD plays 
A

Metabolite Sets Enrichment Overview

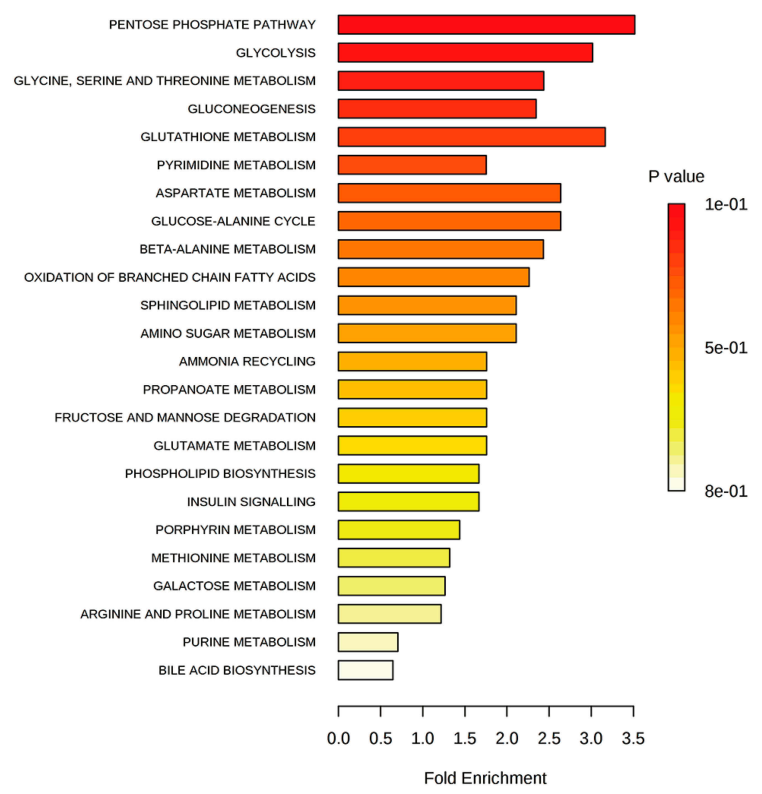

C

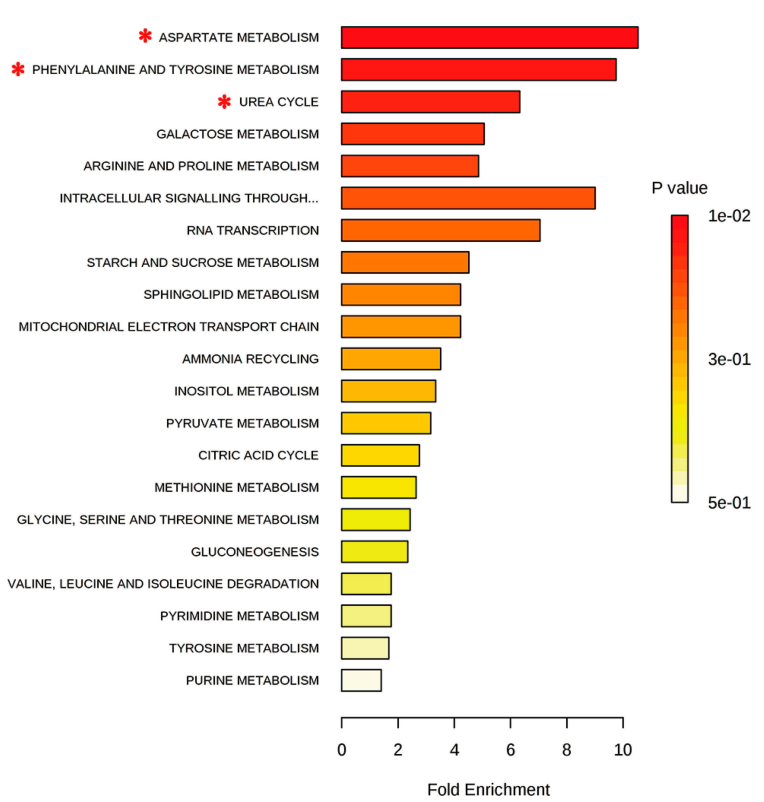

B

Overview of Pathway Analysis

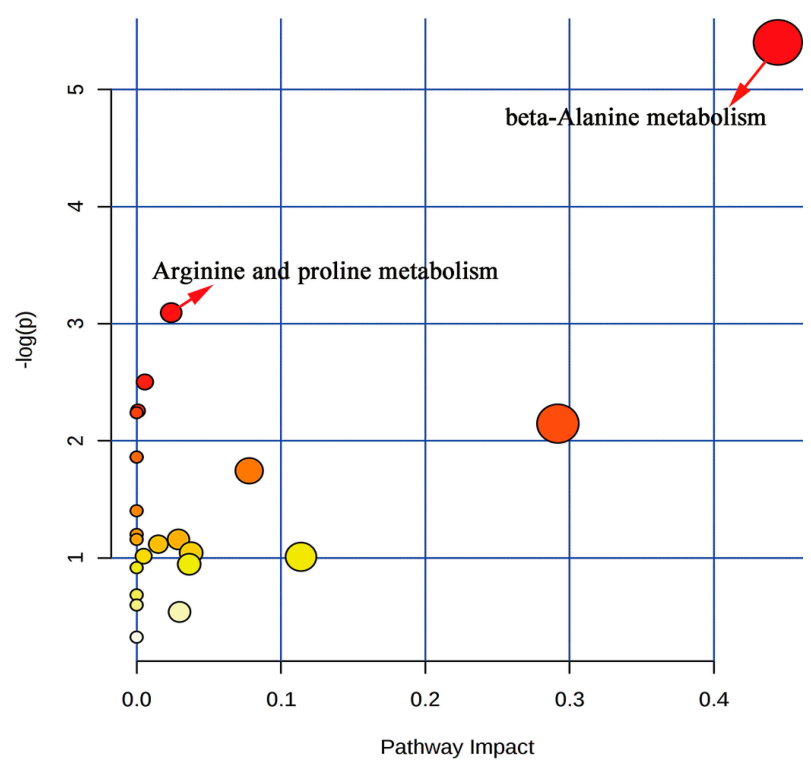

D

Overview of Pathway Analysis

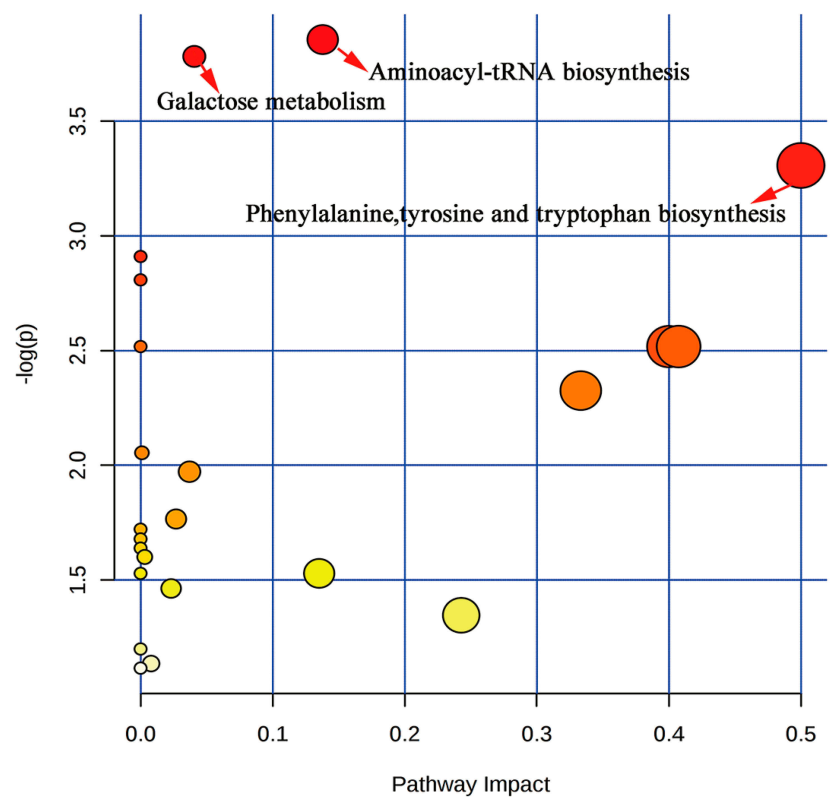

Figure 5 Significant biological functions (A) and pathways (B) of the differential metabolites in the HP associated with DG treatment. Significant biological functions (C) and pathways (D) of the differential metabolites in the PFC associated with DG treatment. *False discovery rate (FDR) $<0.05 ; \rightarrow$ FDR $<0.05$ and impact value $>0$.

a critical role in the tryptophan-NAD pathway. Tryptophan is also involved in the production of serotonin by tryptophan hydroxylase and 5-hydroxytryptophan decarboxylase. ${ }^{55}$ Mono(2-ethylhexyl)phthalate may increase the production of serotonin through the 5-hydroxytryptophan decarboxylase pathway by inhibiting the tryptophan-NAD pathway. Serotonin is a neurotransmitter that is involved in hedonic emotions. Antidepressants such as fluoxetine work by increasing levels of serotonin in the brain. ${ }^{56}$ People with low serotonin levels are more likely to experience depression, impulsive behavior, alcoholism, suicide, aggression and violence. ${ }^{57}$ Previous studies on venlafaxine also found increased levels of mono(2-ethylhexyl)phthalate in the HP. ${ }^{58}$ Therefore, DG may exert an antidepressant action by upregulating mono(2-ethylhexyl)phthalate to increase serotonin levels in the HP and PFC. 
A

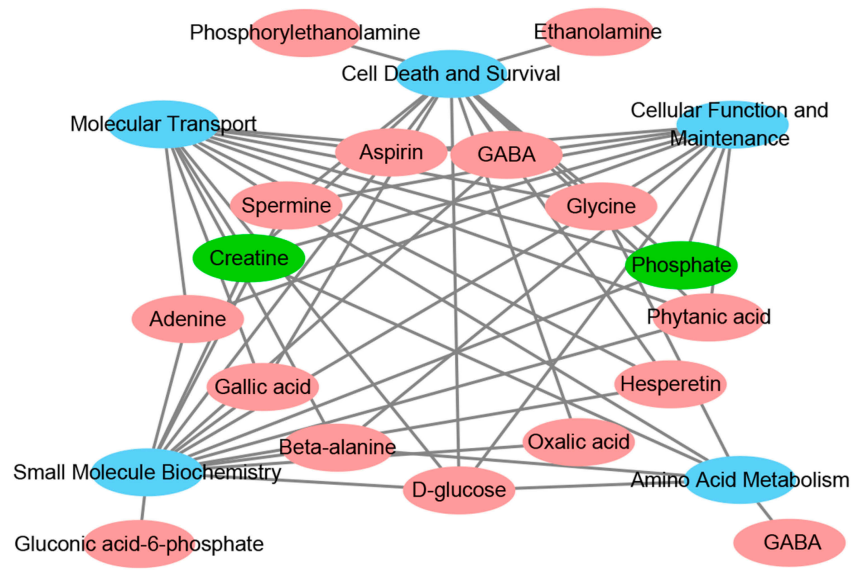

B

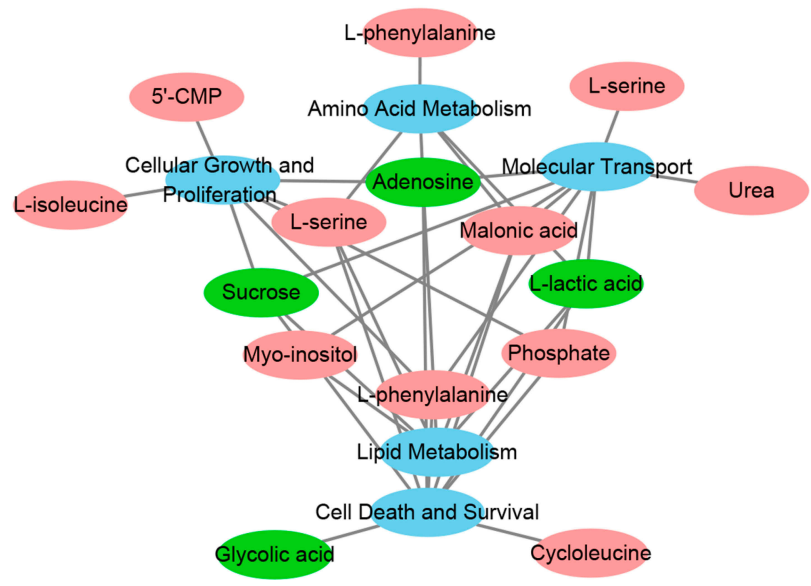

Figure 6 (A) Overview of molecular and cellular functions of the differential metabolites in the hippocampus following 4 weeks of DG treatment, according to the IPA database. (B) Overview of molecular and cellular functions of the differential metabolites in the PFC following 4 weeks of DG treatment, according to the IPA database.

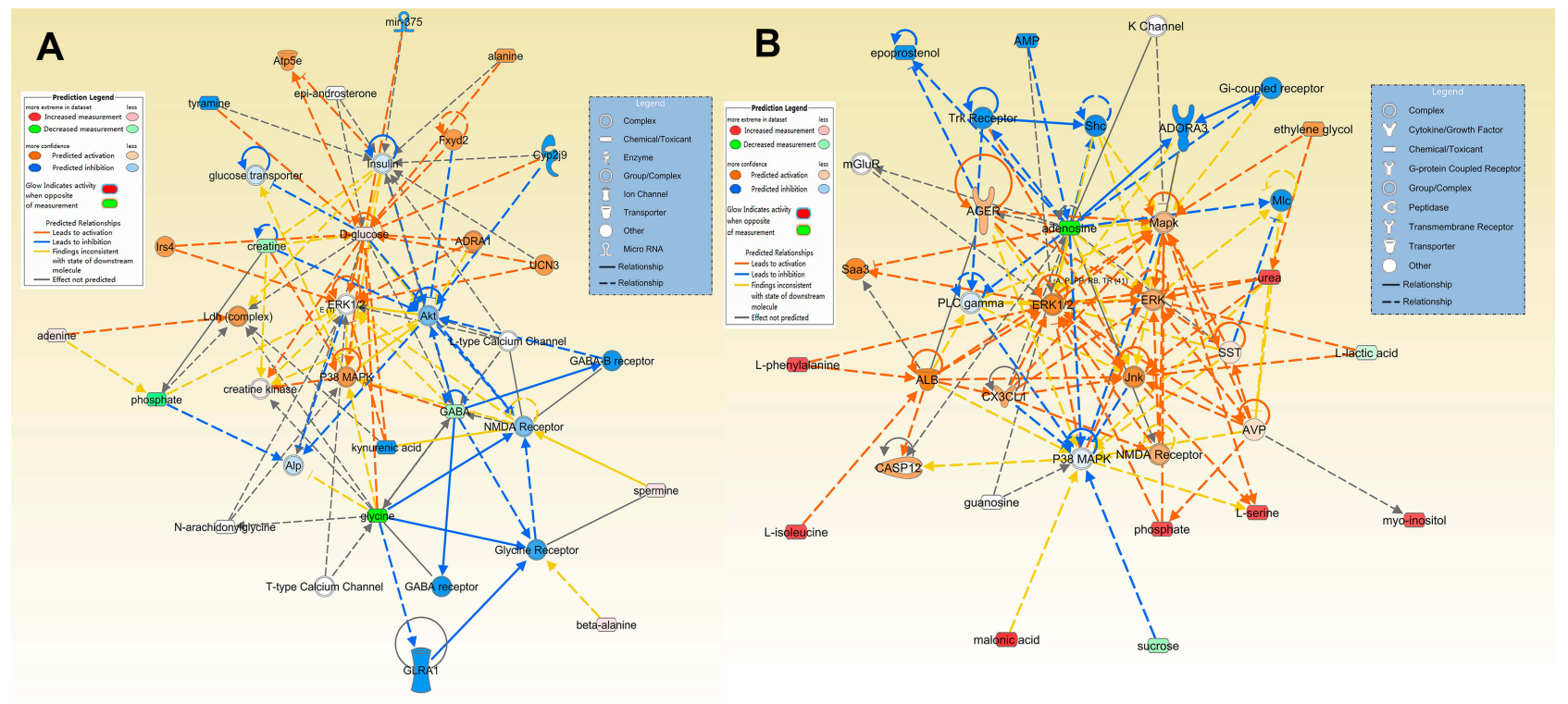

Figure 7 Predicted biological functions (z-score $>2$ or z-score $<-2$ ) associated with DG treatment identified by ingenuity pathways analysis. (A) Networks associated with DG treatment in the HP. (B) Networks associated with DG treatment in the PFC.

Through the IPA metabolite-protein network interaction prediction and KEGG pathway analysis, the NT3-TrkA and Ras-MAPK pathways were found to be closely related to the HP and PFC. Nt3-TrkA is a signaling pathway associated with the neurotrophin family, and plays an important role in axonal growth towards their target. ${ }^{14,15,59}$ NT3 is trafficked mainly through the constitutive secretory pathway in neurons and neuroendocrine cells. ${ }^{60}$ Studies have shown that NT3 is associated with neurodegenerative diseases (eg, Alzheimer's) ${ }^{61,62}$ and psychiatric diseases (eg, depression and suicide). ${ }^{20}$ The Trk receptors are high-affinity tyrosine kinase receptors that preferentially bind particular neurotrophins. ${ }^{63}$ TrkA is one of the receptors for NT3, which is highly expressed in cholinergic neurons in the central nervous system. ${ }^{64}$ NT3-TrkA-dependent axonal growth is associated with activation of the Ras-MAPK signaling pathway. ${ }^{18}$ MAPKs are highly conserved serine/threonine protein kinases that play a role in many cellular processes, such as proliferation, differentiation, stress response, apoptosis and survival. ${ }^{65}$ Ras and Raf are important core proteins in the Ras-MAPK pathway. The RasMAPK signaling pathway plays a key role in neuronal 
A

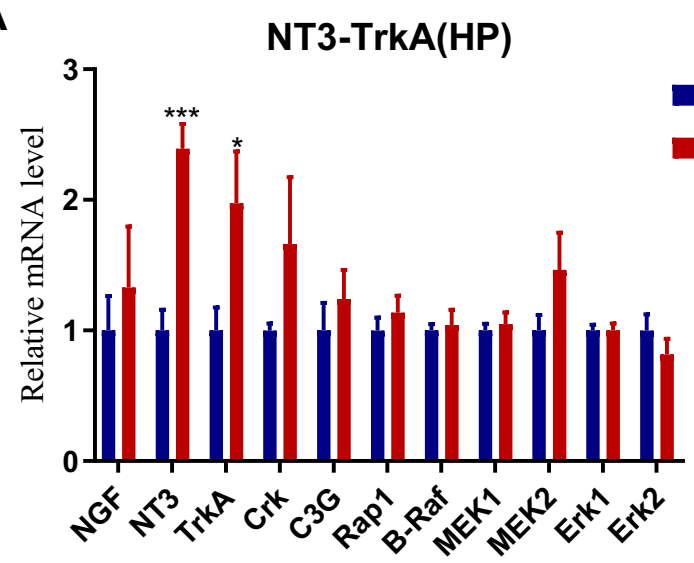

C

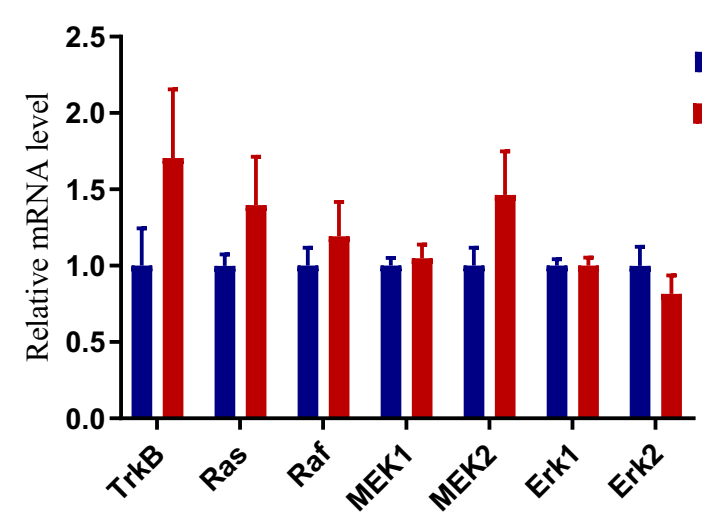

B

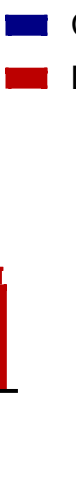

NT3-TrkA(PFC)

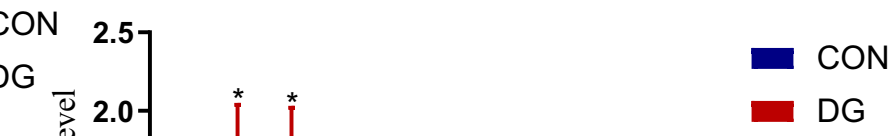

Figure 8 The expression levels of genes related to the NT3-TrkA pathway in the HP (A) and the PFC (B). The expression levels of genes related to the Ras-MAPK pathway in the HP (C) and the PFC (D). Compared with the CON group, the expression levels of NT3 and TrkA in the DG group were significantly increased in the HP (A) and the PFC (B). ${ }^{*} p<0.05, * * * p 0.001$.

plasticity, and it is closely related to the pathogenesis and treatment of depression. ${ }^{22,23,66}$ In our study, the mRNA expression levels of NT3 and TrkA were significantly upregulated in the HP and PFC. However, there was no significant changes in the Ras-MAPK pathway. Western blotting showed that Raf, NT3, Ras and Trk A were significantly upregulated in the HP, while only TrkA was significantly upregulated in the PFC. This suggests that DGs may exert their antidepressant-like effect by activating the NT3-TrkA and Ras-MAPK pathways in the HP (Figure 10).

Pearson correlation analysis between depressionrelated behaviors and genes and differential metabolites in the HP and PFC (Figure 11) revealed no significant differences between the HP and the PFC, with significant brain region specificity. In the OFT, the number of entries in the central region was negatively correlated with the downregulated metabolites (GABA and phosphate) in the
$\mathrm{HP}$, and the distance traveled in the central region was negatively correlated with the upregulated metabolites (adenine in the HP and urea in the PFC). In the EPM, the number of entries in the open arm was positively correlated with the downregulated metabolites (glycine) in the HP and with upregulated metabolites (myoinositol) in the PFC. Ras was positively correlated with upregulated metabolites (spermine) in the HP. In the PFC, Ras was negatively correlated with the upregulated metabolites (L-phenylalanine), and NT3 was positively correlated with the downregulated metabolites (sucrose) and negatively correlated with the upregulated metabolites (L-serine). Raf was positively correlated with the downregulated metabolites (adenosine) in the PFC. Except for the upregulation of L-phenylalanine in the PFC and upregulation of beta-alanine in the HP, which were negatively correlated, the upregulated metabolites in the PFC were 
A

$$
\text { CON }
$$

DG

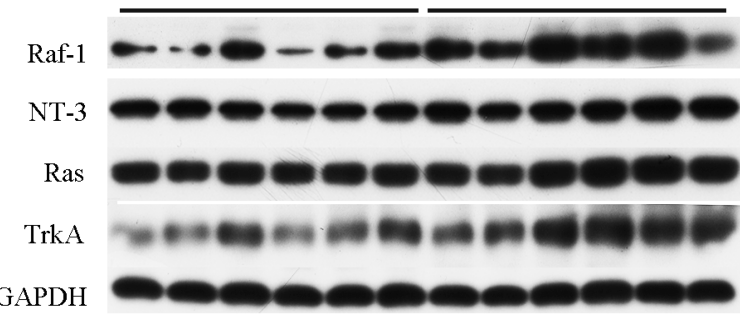

Raf-1-HP

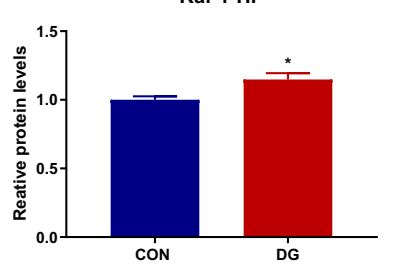

Ras-HP

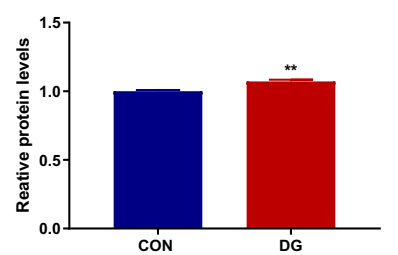

NT3-HP

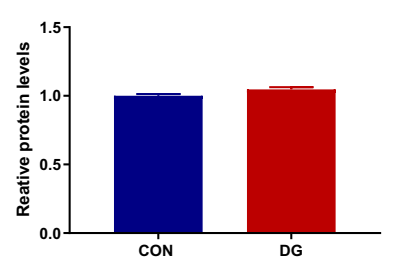

TrkA-HP

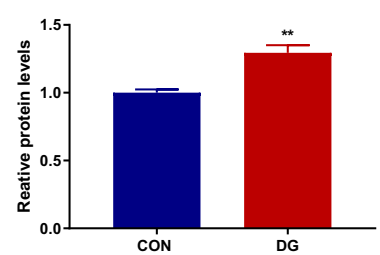

B

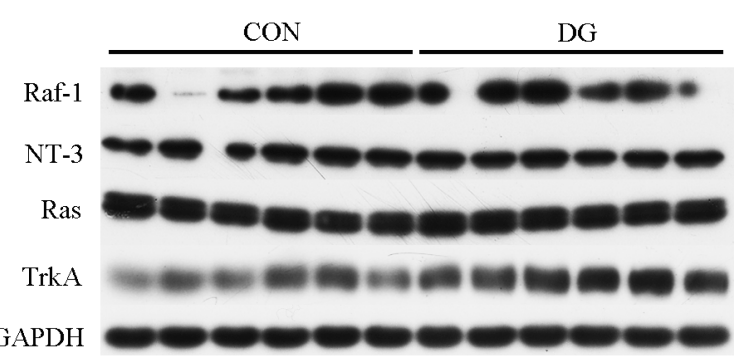

Raf-1-PFC
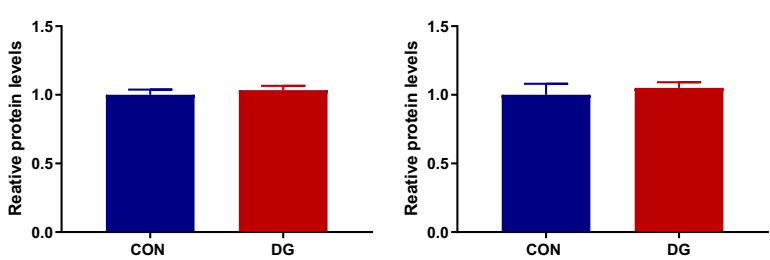

Ras-PFC
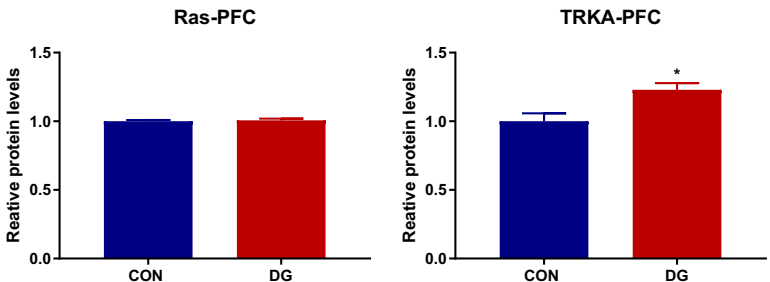

Figure 9 The candidate proteins selected for Western blot analysis. In the DG group, compared with the CON group, the expression levels of Raf, NT3, Ras and TrkA were significantly increased in the HP (A), and the expression levels of TrkA were significantly increased in the PFC (B).*p $<0.05$, **p $<0.0$ I.

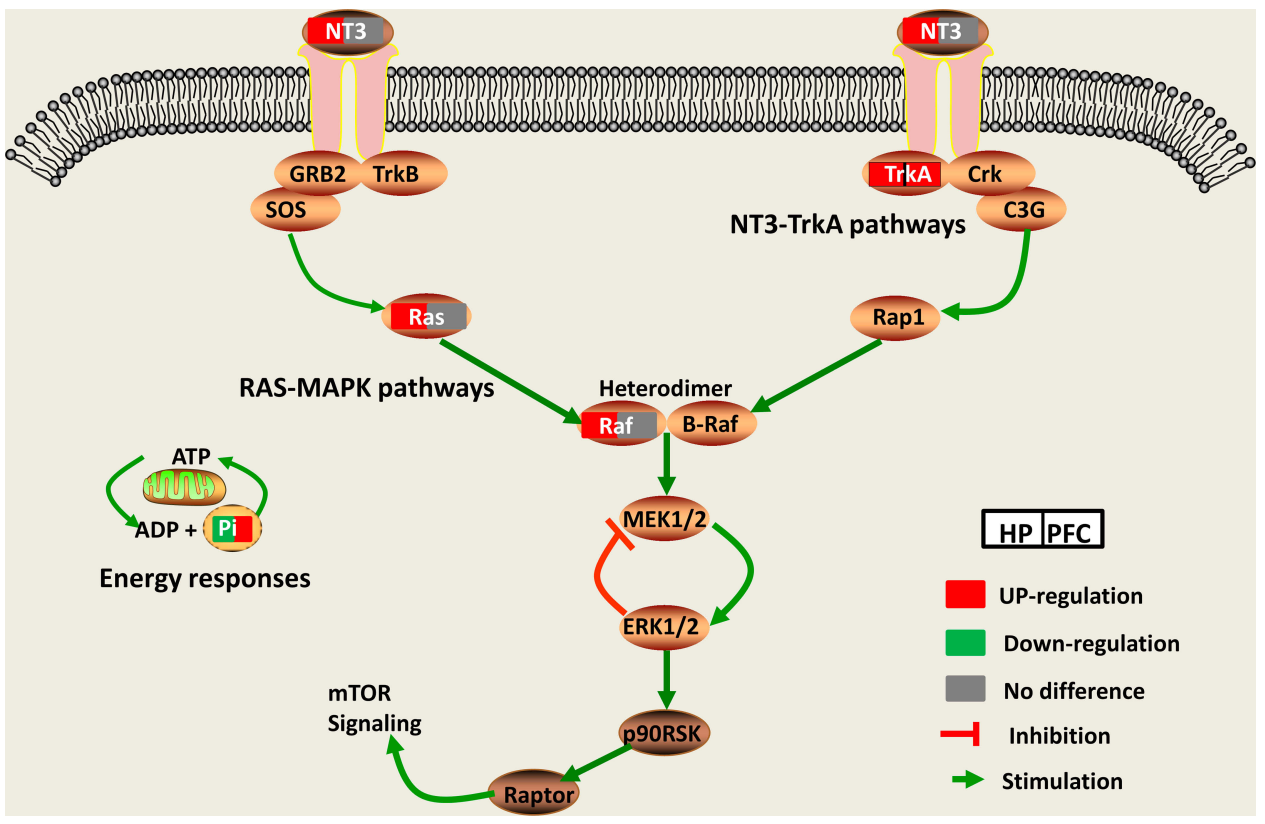

Figure 10 The Ras-MAPK and NT3-TrkA pathways play important roles in the antidepressant effect of DG in the HP and PFC.

positively correlated with the downregulated metabolites in the HP, while the downregulated metabolites in the PFC were negatively correlated with the downregulated metabolites in the HP. Thus, the Pearson analysis revealed substantial brain region specificity in behavioral, gene expression and metabolic changes. 


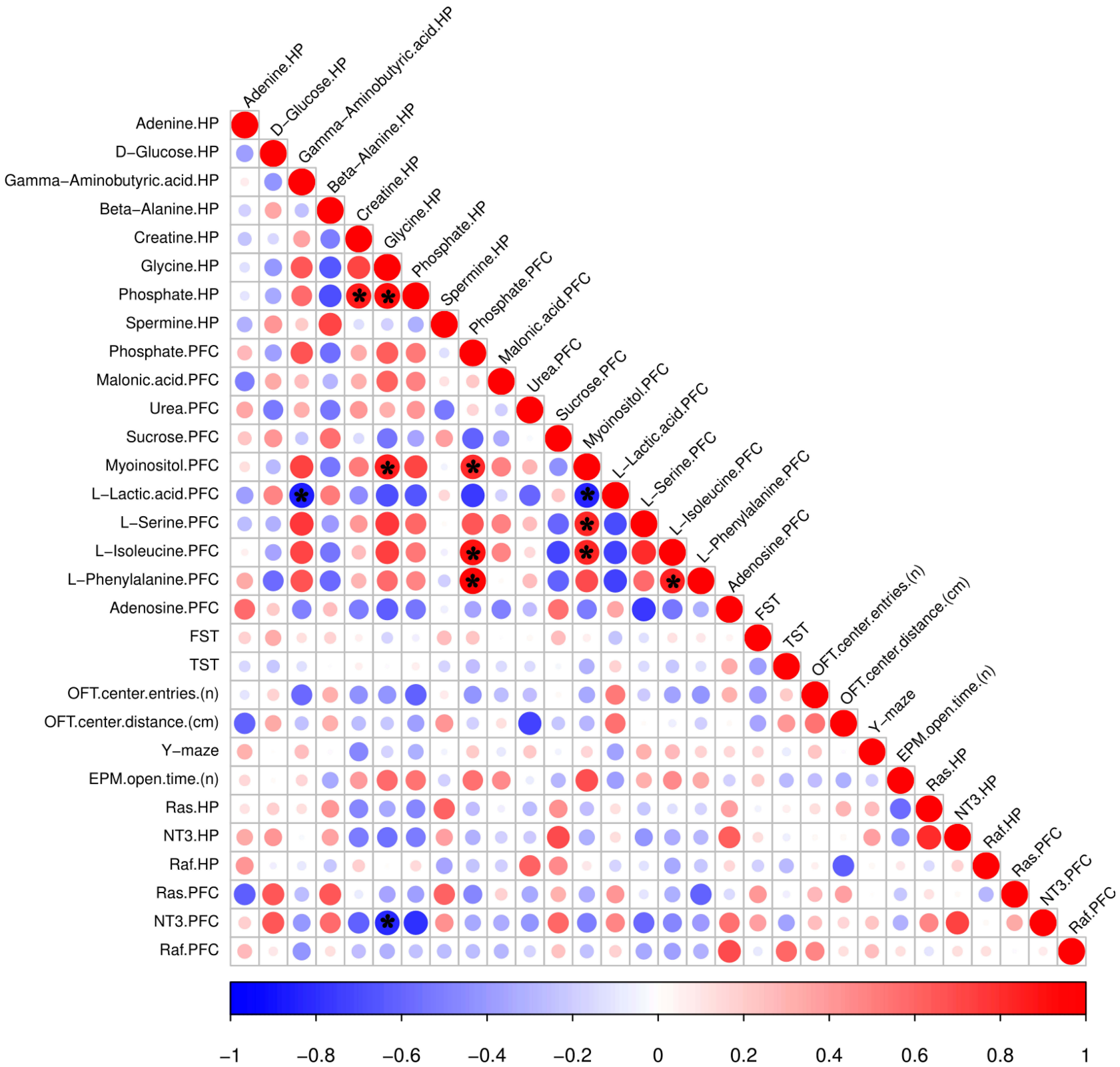

Figure II Metabolites correlated with behavior and gene expression. The color and size of the circles in the matrix indicate the level of correlation; blue represents a negative correlation, while red represents a positive correlation. $*_{p}<0.05$.

\section{Conclusion}

Our findings show that chronic DG treatment effectively alleviates despair and anxiety-like behaviors in rats without side effects. The antidepressant effect of DGs was associated with brain region-specific changes in the levels of metabolites and proteins in the hippocampus and prefrontal cortex. The correlation analysis suggested that the antidepressant effect of DG is related to the levels of GABA, phosphate and spermine in the hippocampus and myoinositol in the prefrontal cortex. In addition, DG appears to exert its antidepressant effect mainly by activating the neurotrophin-related NT3-TrkA pathway and the neural plasticity-related Ras-MAPK pathway in the hippocampus. Our findings provide novel insight into the antidepressant mechanism of action of DG and provide new molecular targets for the development of effective therapeutic strategies for depression.

\section{Acknowledgments}

This work was supported by the National Key R\&D Program of China (Grant No. 2017YFA0505700), the Non-profit Central Research Institute Fund of Chinese 
Academy of Medical Sciences (No.2019PT320002), the National Natural Science Foundation of China (Grant No. 81901398), the National Key Program International Cooperation Project (No. 81820108015) and the Natural Science Foundation of Chongqing, China (Grant No. cstc2019jcyj-msxmX0025).

\section{Disclosure}

The authors declare that they have no conflicts of interest in this work.

\section{References}

1. Duman RS, Monteggia LM. A neurotrophic model for stress-related mood disorders. Biol Psychiatry. 2006;59(12):1116-1127. doi:10.10 16/j.biopsych.2006.02.013

2. Kessler RC, Berglund P, Demler O, et al. The epidemiology of major depressive disorder: results from the National Comorbidity Survey Replication (NCS-R). JAMA. 2003;289(23):3095-3105. doi:10.1001/ jama.289.23.3095

3. Cipriani A, Zhou X, Del Giovane C, et al. Comparative efficacy and tolerability of antidepressants for major depressive disorder in children and adolescents: a network meta-analysis. Lancet. 2016;388 (10047):881-890. doi:10.1016/S0140-6736(16)30385-3

4. Xia CY, Wang ZZ, Yamakuni T, Chen NH. A novel mechanism of depression: role for connexins. Eur Neuropsychopharmacol. 2018;28 (4):483-498. doi:10.1016/j.euroneuro.2018.01.009

5. Zhang Y, Bi X, Adebiyi O, et al. Venlafaxine improves the cognitive impairment and depression-like behaviors in a cuprizone mouse model by alleviating demyelination and neuroinflammation in the brain. Front Pharmacol. 2019;10:332. doi:10.3389/fphar.2019.00332

6. Yang SJ, Song ZJ, Wang XC, Zhang ZR, Wu SB, Zhu GQ. Curculigoside facilitates fear extinction and prevents depression-like behaviors in a mouse learned helplessness model through increasing hippocampal BDNF. Acta Pharmacol Sin. 2019;40(10):1269-1278. doi:10.1038/s41401-019-0238-4

7. Su WJ, Cao ZY, Jiang CL. [Inflammatory mechanism of depression and its new strategy for diagnosis and treatment]. Sheng Li Xue Bao. 2017;69(5):715-722. Chinese.

8. Hu Q, Shen P, Bai S, et al. Metabolite-related antidepressant action of diterpene ginkgolides in the prefrontal cortex. Neuropsychiatr Dis Treat. 2018;14:999-1011. doi:10.2147/NDT.S161351

9. Bai S, Zhang X, Chen Z, et al. Insight into the metabolic mechanism of Diterpene Ginkgolides on antidepressant effects for attenuating behavioural deficits compared with venlafaxine. Sci Rep. 2017;7 (1):9591. doi:10.1038/s41598-017-10391-1

10. Liang Z, Bai S, Shen P, et al. GC-MS-based metabolomic study on the antidepressant-like effects of diterpene ginkgolides in mouse hippocampus. Behav Brain Res. 2016;314:116-124. doi:10.1016/j. bbr.2016.08.001

11. Kennedy DO, Scholey AB, Wesnes KA. Differential, dose dependent changes in cognitive performance following acute administration of a Ginkgo biloba/Panax ginseng combination to healthy young volunteers. Nutr Neurosci. 2001;4(5):399-412. doi:10.1080/102841 5X.2001.11747376

12. Menku A, Koc RK, Tayfur V, Saraymen R, Narin F, Akdemir H. Effects of mexiletine, ginkgo biloba extract (EGb 761), and their combination on experimental head injury. Neurosurg Rev. 2003;26 (4):288-291.

13. Hua J, Yin N, Yang B, et al. Ginkgolide B and bilobalide ameliorate neural cell apoptosis in alpha-synuclein aggregates. Biomed Pharmacother. 2017;96:792-797. doi:10.1016/j.biopha.2017.10.050
14. Bagal SK, Andrews M, Bechle BM, et al. Discovery of potent, selective, and peripherally restricted pan-trk kinase inhibitors for the treatment of pain. J Med Chem. 2018;61(15):6779-6800. doi:10. 1021/acs.jmedchem. 8 b00633

15. Keeler AB, Suo D, Park J, Deppmann CD. Delineating neurotrophin-3 dependent signaling pathways underlying sympathetic axon growth along intermediate targets. Mol Cell Neurosci. 2017; 82:66-75. doi:10.1016/j.men.2017.04.011

16. Wang JQ, Tang Q, Parelkar NK, et al. Glutamate signaling to Ras-MAPK in striatal neurons: mechanisms for inducible gene expression and plasticity. Mol Neurobiol. 2004;29(1):1-14. doi:10.1385/MN:29:1

17. Rezatabar S, Karimian A, Rameshknia V, et al. RAS/MAPK signaling functions in oxidative stress, DNA damage response and cancer progression. J Cell Physiol. 2019;234(9):14951-14965. doi:10.1002/jcp. v234.9

18. Suo D, Park J, Harrington AW, Zweifel LS, Mihalas S, Deppmann CD. Coronin-1 is a neurotrophin endosomal effector that is required for developmental competition for survival. Nat Neurosci. 2014;17(1):36-45. doi:10.1038/nn.3593

19. Nakamura T, Sanokawa R, Sasaki Y, Ayusawa D, Oishi M, Mori N. N-Shc: a neural-specific adapter molecule that mediates signaling from neurotrophin/Trk to Ras/MAPK pathway. Oncogene. 1996;13 (6):1111-1121.

20. Dwivedi Y. Brain-derived neurotrophic factor: role in depression and suicide. Neuropsychiatr Dis Treat. 2009;5:433-449. doi:10.2147/NDT

21. Sheldrick A, Camara S, Ilieva M, Riederer P, Michel TM. Brainderived neurotrophic factor (BDNF) and neurotrophin 3 (NT3) levels in post-mortem brain tissue from patients with depression compared to healthy individuals - a proof of concept study. Eur Psychiatry. 2017;46:65-71. doi:10.1016/j.eurpsy.2017.06.009

22. Jiang P, Zhu T, Xia Z, et al. Inhibition of MAPK/ERK signaling blocks hippocampal neurogenesis and impairs cognitive performance in prenatally infected neonatal rats. Eur Arch Psychiatry Clin Neurosci. 2015;265(6):497-509. doi:10.1007/s00406-015-0588-y

23. Sanchez-Ortiz E, Cho W, Nazarenko I, Mo W, Chen J, Parada LF. NF1 regulation of RAS/ERK signaling is required for appropriate granule neuron progenitor expansion and migration in cerebellar development. Genes Dev. 2014;28(21):2407-2420. doi:10.1101/gad. 246603.114

24. Malberg JE, Eisch AJ, Nestler EJ, Duman RS. Chronic antidepressant treatment increases neurogenesis in adult rat hippocampus. $J$ Neurosci. 2000;20(24):9104-9110. doi:10.1523/JNEUROSCI.2024-09104.2000

25. Tanapat P, Hastings NB, Rydel TA, Galea LA, Gould E. Exposure to fox odor inhibits cell proliferation in the hippocampus of adult rats via an adrenal hormone-dependent mechanism. J Comp Neurol. 2001;437(4):496-504. doi:10.1002/cne.1297

26. Murray EA, Wise SP. Interactions between orbital prefrontal cortex and amygdala: advanced cognition, learned responses and instinctive behaviors. Curr Opin Neurobiol. 2010;20(2):212-220. doi:10.1016/j. conb.2010.02.001

27. Cardinal RN, Parkinson JA, Hall J, Everitt BJ. Emotion and motivation: the role of the amygdala, ventral striatum, and prefrontal cortex. Neurosci Biobehav Rev. 2002;26(3):321-352. doi:10.1016/S01497634(02)00007-6

28. Mao Q, Gong X, Zhou C, et al. Up-regulation of SIRT6 in the hippocampus induced rats with depression-like behavior via the block Akt/GSK3beta signaling pathway. Behav Brain Res. 2017;3 23:38-46. doi:10.1016/j.bbr.2017.01.035

29. Han X, Shao W, Liu Z, et al. iTRAQ-based quantitative analysis of hippocampal postsynaptic density-associated proteins in a rat chronic mild stress model of depression. Neuroscience. 2015;298:220-292. doi:10.1016/j.neuroscience.2015.04.006

30. Chen G, Yang D, Yang Y, et al. Amino acid metabolic dysfunction revealed in the prefrontal cortex of a rat model of depression. Behav Brain Res. 2015;278:286-292. doi:10.1016/j.bbr.2014.05.027 
31. Wang W, Guo H, Zhang SX, et al. Targeted metabolomic pathway analysis and validation revealed glutamatergic disorder in the prefrontal cortex among the chronic social defeat stress mice model of depression. J Proteome Res. 2016;15(10):3784-3792. doi:10.1021/ acs.jproteome.6b00577

32. Chermat R, Thierry B, Mico JA, Steru L, Simon P. Adaptation of the tail suspension test to the rat. J Pharmacol. 1986;17(3):348-350.

33. Naqvi F, Haider S, Batool Z, Perveen T, Haleem DJ. Sub-chronic exposure to noise affects locomotor activity and produces anxiogenic and depressive like behavior in rats. Pharmacol Rep. 2012;64 (1):64-69. doi:10.1016/S1734-1140(12)70731-4

34. Belozertseva IV, Kos T, Popik P, Danysz W, Bespalov AY. Antidepressantlike effects of mGluR1 and mGluR5 antagonists in the rat forced swim and the mouse tail suspension tests. Eur Neuropsychopharmacol. 2007;17 (3):172-179. doi:10.1016/j.euroneuro.2006.03.002

35. Hoban AE, Moloney RD, Golubeva AV, et al. Behavioural and neurochemical consequences of chronic gut microbiota depletion during adulthood in the rat. Neuroscience. 2016;339:463-477. doi:10.1016/j.neuroscience.2016.10.003

36. Zheng P, Zeng B, Liu M, et al. The gut microbiome from patients with schizophrenia modulates the glutamate-glutamine-GABA cycle and schizophrenia-relevant behaviors in mice. Sci Adv. 2019;5(2): eaau8317. doi:10.1126/sciadv.aau8317

37. Mineur YS, Picciotto MR, Sanacora G. Antidepressant-like effects of ceftriaxone in male C57BL/6J mice. Biol Psychiatry. 2007;61 (2):250-252. doi:10.1016/j.biopsych.2006.04.037

38. Zheng P, Zeng B, Zhou C, et al. Gut microbiome remodeling induces depressive-like behaviors through a pathway mediated by the host's metabolism. Mol Psychiatry. 2016;21(6):786-796. doi:10.1038/ mp.2016.44

39. Luedemann A, Strassburg K, Erban A, Kopka J. TagFinder for the quantitative analysis of gas chromatography-mass spectrometry (GCMS)-based metabolite profiling experiments. Bioinformatics. 2008;24 (5):732-737. doi:10.1093/bioinformatics/btn023

40. Xia J, Sinelnikov IV, Han B, Wishart DS. MetaboAnalyst 3.0-making metabolomics more meaningful. Nucleic Acids Res. 2015;43(W1) W251-W257. doi:10.1093/nar/gkv380

41. Kramer A, Green J, Pollard J Jr, Tugendreich S. Causal analysis approaches in ingenuity pathway analysis. Bioinformatics. 2014;30 (4):523-530. doi:10.1093/bioinformatics/btt703

42. Burokas A, Martin-Garcia E, Espinosa-Carrasco J, et al. Extinction and reinstatement of an operant responding maintained by food in different models of obesity. Addict Biol. 2018;23(2):544-555. doi:10.1111/adb.2018.23.issue-2

43. Desbonnet L, Clarke G, Shanahan F, Dinan TG, Cryan JF. Microbiota is essential for social development in the mouse. Mol Psychiatry. 2014;19(2):146-148. doi:10.1038/mp.2013.65

44. Kelly J. Thinking outside (and inside) the box. how Cranberry Township, Pa., designed a safer ambulance. JEMS. 2016;41(10):24-28.

45. Tramullas M, Dinan TG, Cryan JF. Chronic psychosocial stress induces visceral hyperalgesia in mice. Stress. 2012;15(3):281-292. doi:10.3109/10253890.2011.622816

46. Ansari R, Mahta A, Mallack E, Luo JJ. Hyperhomocysteinemia and neurologic disorders: a review. J Clin Neurol. 2014;10(4):281-288. doi:10.3988/jen.2014.10.4.281

47. Reher M, Fuhrer T, Bott M, Schonheit P. The nonphosphorylative Entner-Doudoroff pathway in the thermoacidophilic euryarchaeon Picrophilus torridus involves a novel 2-keto-3-deoxygluconatespecific aldolase. J Bacteriol. 2010;192(4):964-974. doi:10.1128/ JB.01281-09

48. Reher M, Schonheit P. Glyceraldehyde dehydrogenases from the thermoacidophilic euryarchaeota Picrophilus torridus and Thermoplasma acidophilum, key enzymes of the non-phosphorylative Entner-Doudoroff pathway, constitute a novel enzyme family within the aldehyde dehydrogenase superfamily. FEBS Lett. 2006;580(5):1198-1204. doi:10.1016/j. febslet.2006.01.029
49. Belanger M, Allaman I, Magistretti PJ. Brain energy metabolism: focus on astrocyte-neuron metabolic cooperation. Cell Metab. 2011;14(6):724-738. doi:10.1016/j.cmet.2011.08.016

50. Harper DG, Joe EB, Jensen JE, Ravichandran C, Forester BP. Brain levels of high-energy phosphate metabolites and executive function in geriatric depression. Int $J$ Geriatr Psychiatry. 2016;31(11):12 41-1249. doi:10.1002/gps.v31.11

51. Pimontel MA, Culang-Reinlieb ME, Morimoto SS, Sneed JR. Executive dysfunction and treatment response in late-life depression. Int J Geriatr Psychiatry. 2012;27(9):893-899. doi:10.10 02/gps.v27.9

52. Rabinovici GD, Stephens ML, Possin KL. Executive dysfunction. Continuum. 2015;21(3 Behavioral Neurology and Neuropsychiatry):646-659. doi:10.1212/01.CON.0000466658.05156.54

53. David RM, Moore MR, Finney DC, Guest D. Chronic toxicity of di (2-ethylhexyl)phthalate in rats. Toxicol Sci. 2000;55(2):433-443. doi:10.1093/toxsci/55.2.433

54. Fukuwatari T, Ohsaki S, Fukuoka S, Sasaki R, Shibata K. Phthalate esters enhance quinolinate production by inhibiting alpha-amino-beta -carboxymuconate-epsilon-semialdehyde decarboxylase (ACMSD), a key enzyme of the tryptophan pathway. Toxicol Sci. 2004;81 (2):302-308. doi:10.1093/toxsci/kfh204

55. Ara I, Bano S. Citalopram decreases tryptophan 2,3-dioxygenase activity and brain 5-HT turnover in swim stressed rats. Pharmacol Rep. 2012;64(3):558-566. doi:10.1016/S1734-1140(12)70851-4

56. Page ME, Detke MJ, Dalvi A, Kirby LG, Lucki I. Serotonergic mediation of the effects of fluoxetine, but not desipramine, in the rat forced swimming test. Psychopharmacology. 1999;147(2):16 2-167. doi:10.1007/s002130051156

57. Spreux-Varoquaux O, Alvarez JC, Berlin I, et al. Differential abnormalities in plasma 5-HIAA and platelet serotonin concentrations in violent suicide attempters: relationships with impulsivity and depression. Life Sci. 2001;69(6):647-657. doi:10.1016/S0024-3205 (01)01158-4

58. Bai S, Hu Q, Chen Z, et al. Brain region-specific metabolite networks regulate antidepressant effects of venlafaxine. RSC $A d v .2017 ; 7$ (73):46358-46369. doi:10.1039/C7RA08726H

59. Suo D, Park J, Young S, Makita T, Deppmann CD. Coronin-1 and calcium signaling governs sympathetic final target innervation. $J$ Neurosci. 2015;35(9):3893-3902. doi:10.1523/JNEUROSCI.440214.2015

60. Hibbert AP, Morris SJ, Seidah NG, Murphy RA. Neurotrophin-4, alone or heterodimerized with brain-derived neurotrophic factor, is sorted to the constitutive secretory pathway. J Biol Chem. 2003;278 (48):48129-48136. doi:10.1074/jbc.M300961200

61. Schulte-Herbruggen O, Jockers-Scherubl MC, Hellweg R. Neurotrophins: from pathophysiology to treatment in Alzheimer's disease. Curr Alzheimer Res. 2008;5(1):38-44. doi:10.2174/156720 508783884620

62. Saragovi HU, Hamel E, Di Polo A. A neurotrophic rationale for the therapy of neurodegenerative disorders. Curr Alzheimer Res. 2009;6 (5):419-423. doi:10.2174/156720509789207912

63. Prakash Y, Thompson MA, Meuchel L, et al. Neurotrophins in lung health and disease. Expert Rev Respir Med. 2010;4(3):395-411. doi:10.1586/ers.10.29

64. Sanchez-Ortiz E, Yui D, Song D, et al. TrkA gene ablation in basal forebrain results in dysfunction of the cholinergic circuitry. $J$ Neurosci. 2012;32(12):4065-4079. doi:10.1523/JNEUROSCI.63 14-11.2012

65. Arthur JS, Ley SC. Mitogen-activated protein kinases in innate immunity. Nat Rev Immunol. 2013;13(9):679-692. doi:10.1038/ nri3495

66. Denayer E, Ahmed T, Brems H, et al. Spred1 is required for synaptic plasticity and hippocampus-dependent learning. $J$ Neurosci. 2008;28(53):14443-14449. doi:10.1523/JNEUROSCI. 4698-08.2008 


\section{Publish your work in this journal}

Drug Design, Development and Therapy is an international, peerreviewed open-access journal that spans the spectrum of drug design and development through to clinical applications. Clinical outcomes, patient safety, and programs for the development and effective, safe, and sustained use of medicines are a feature of the journal, which has also been accepted for indexing on PubMed Central. The manuscript management system is completely online and includes a very quick and fair peer-review system, which is all easy to use. Visit http://www. dovepress.com/testimonials.php to read real quotes from published authors. 